\title{
Las mariposas (Hesperioidea y Papilionoidea) de Malinalco, Estado de México
}

\author{
The butterflies (Hesperioidea and Papilionoidea) of Malinalco, State of Mexico
}

\author{
Claudia Hernández-Mejía, Jorge Llorente-Bousquets*, Isabel Vargas-Fernández y Armando Luis-Martínez \\ Museo de Zoología Alfonso L. Herrera, Facultad de Ciencias, Universidad Nacional Autónoma de México. Apartado postal 70-399, 04510 México, D. F. \\ *Correspondencia: jlb@hp.fciencias.unam.mx
}

\begin{abstract}
Resumen. Se realizó un estudio sobre la composición, distribución altitudinal y gremios alimentarios de mariposas diurnas en Malinalco, Estado de México. Durante un año se recolectó de manera sistemática en 12 localidades ubicadas ente 1 000 y 2000 m snm, obteniendo un total de 1333 ejemplares, cuya determinación taxonómica permitió generar una lista integrada por 2 superfamilias, 6 familias, 21 subfamilias, 147 géneros y 213 especies, de las cuales 157 son registros nuevos (63 regionales y 94 estatales). La familia más diversa fue Hesperiidae (74 especies), seguida de Nymphalidae (55), Lycaenidae (30), Pieridae (26), Papilionidae (16) y Riodinidae (12). Los géneros mejor representados en cuanto a riqueza de especies fueron Urbanus, Strymon, Emesis y Chlosyne. Con base en la curva de acumulación de especies, empleando el modelo de Clench, se recolectó el $81 \%$ del valor teórico esperado. A la lista generada se agregó información de la base de datos Mariposa del Museo de Zoología Alfonso L. Herrera de la Facultad de Ciencias, UNAM, con lo que se alcanzó un total de 240 especies registradas para el municipio. En época de sequía, la mayor diversidad de especies se concentró en mayo (102), mes en que se inicia el periodo de lluvias; en este último, en julio, se observó el vuelo de 62 especies, que en agosto disminuyeron a 42. Respecto a la distribución altitudinal, el 31\% de las especies establece sus poblaciones en sitios inferiores a $1400 \mathrm{~m}$. Con base en las preferencias de alimentación y en la disponibilidad de los recursos aprovechables por los imagos, se determinaron 3 gremios alimentarios: nectarívoros (54\%), hidrófilos (2\%) y acimófagos (3\%).
\end{abstract}

Palabras clave: México, Hesperioidea, Papilionoidea, distribución altitudinal, gremios alimentarios.

\begin{abstract}
The species composition, altitudinal distribution and adult food resources of the butterfly fauna of Malinalco, Mexico State, were studied. Over the course of one year, systematic collections were carried out at 12 localities between 1000 and $2000 \mathrm{~m}$ of elevation, resulting in 1333 specimens, which represent 2 superfamilies, 6 families, 21 subfamilies, 147 genera and 213 species, of which 157 are new records (63 new regional records, 94 new state records). The most diverse family was Hesperiidae (74 species), followed by Nymphalidae (55 species), Lycaenidae ( 30 species), Pieridae (26 species), Papilionidae (16 species) and Riodinidae (12 species). The best-represented genera in terms of species richness were Urbanus, Strymon, Emesis and Chlosyne. Based on a fitted species accumulation curve, following Clench's model, $81 \%$ of the number of species expected to occur were actually collected. The list of species generated from this fieldwork was increased with additional data from the Mariposa database at the Museo de Zoología Alfonso L. Herrera, Facultad de Ciencias, UNAM, for a total of 240 species recorded from the Municipality. The greatest diversity of species (102) was found in May, coinciding with the beginning of the annual rainy season, followed by 62 species in July, and 42 species in August. Thirty one percent of the species were only found below $1400 \mathrm{~m}$. Based on feeding preferences and available food resources of adults, three feeding guilds were determined: nectar-feeding (54\%), mud-puddling (2\%) and yeast-feeding $(3 \%)$.
\end{abstract}

Key words: Mexico, Hesperioidea, Papilionoidea, altitudinal distribution, feeding guilds.

\section{Introducción}

Malinalco se encuentra en una región de considerable diversidad biológica; su ubicación geográfica, fisiografía y clima favorecen el establecimiento de diversas comunidades vegetales, en especial la selva baja caducifolia, vegetación que predomina en la entidad. En la cabecera municipal y

Recibido: 09 enero 2007; aceptado: 24 mayo 2007 en los ejidos hay plantas ornamentales y extensos huertos frutales, hábitats propicios para mantener la diversidad de mariposas, al encontrar en ellos las plantas de alimentación para las fases larval y adulta, así como las condiciones para su desarrollo. A pesar de esto, los estudios sobre los Rhopalocera en la región son prácticamente nulos.

Hay publicaciones que hacen referencia a especies recolectadas, cuyo enfoque ha sido el estudio sobre la morfología o taxonomía de determinada especie o grupo 
supraespecífico, o simplemente la búsqueda de especies raras o de interés para las colecciones, entre otras, los trabajos de Vázquez (1951), Miller (1974, 1978), Adams (1983), Jenkins (1983, 1986), Beutelspacher y Howe (1984) y Kendall y McGuire (1984).

El primer trabajo lepidopterofaunístico que se realizó en el municipio fue el de Guzmán (1976), quien citó 45 especies para la localidad de Chalma (13 hespéridas, 3 papiliónidas, 8 piéridas, 3 licénidas y 18 ninfálidas). La información recopilada a partir de la base de datos Mariposa (LuisMartínez et al., 2005) indica que hay 148 papilionoideas y hesperioideas registradas para Malinalco, 100 de ellas se encuentran en colecciones nacionales e internacionales (Cuadro 1), y otras están citadas en la bibliografía. Al no existir estudios rigurosos y estandarizados (Pozo et al., 2005) sobre mariposas en la región, se realizó una recolecta sistemática durante un año (2001-2002) en 12 localidades seleccionadas previamente, con la finalidad de conocer la composición específica de las superfamilias Hesperioidea y Papilionoidea. Se consideraron también algunos aspectos biológicos y ecológicos de las especies para conocer los gremios alimentarios de los imagos y la distribución que ocupan en un gradiente altitudinal. En su mayoría, el material recolectado se depositó en la Colección Nacional de Insectos del Instituto de Biología, UNAM (IBUNAM), y una muestra en el Museo Universitario Luis Mario Schneider Z. ubicado en Malinalco.

Generalidades geográficas. El municipio de Malinalco tiene una superficie de $266.17 \mathrm{~km}^{2}$, se ubica al sureste del Estado de México, entre los paralelos $19^{\circ} 01^{\prime} 58^{\prime \prime}$ -

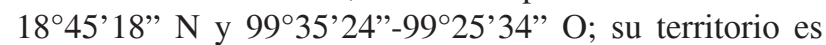
parte de la Sierra Madre del Sur, el Eje volcánico y la Cuenca del Balsas, esencialmente de las sierras y valles guerrerenses (INEGI, 1995) (Fig. 1). Pertenece a la región hidrológica Río Balsas y a la Cuenca Río Grande de

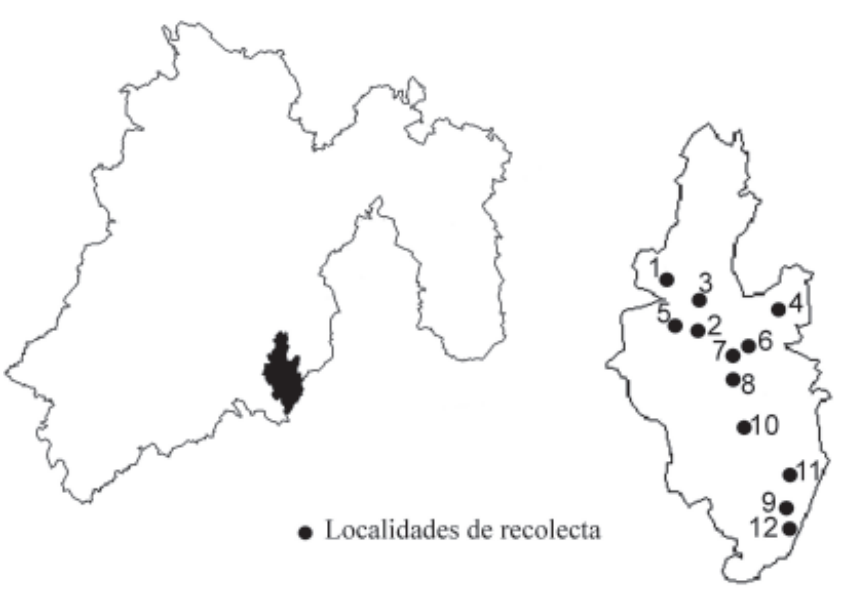

Figura 1. Ubicación geográfica de Malinalco, Estado de México.

Amacuzac (Reyes, 1992); los ríos más importantes son el Chalma y el Tlaxipehualco. El área está acotada entre los 850 y 2600 m snm (Schneider, 1999). Presenta 2 tipos de clima $(\mathrm{A}) \mathrm{C}\left(\mathrm{w}_{1}\right)(\mathrm{w})\left(\mathrm{i}^{\prime}\right) \mathrm{g}$ y $\mathrm{Aw}_{1}(\mathrm{w})\left(\mathrm{i}^{\prime}\right) \mathrm{g}$, la temperatura más alta $\left(22.6^{\circ} \mathrm{C}\right)$ se registra en abril y la más baja $\left(17.6{ }^{\circ} \mathrm{C}\right)$ en enero; el régimen de lluvias es de verano, la precipitación media anual es de $1188.1 \mathrm{~mm}$, con el mes más húmedo en julio y el más seco en febrero (García, 1988) (Fig. 2).

La vegetación predominante es de selva baja caducifolia (SBC), las especies arbóreas más comunes son: Ficus petiolaris, Bursera morelensis, B. pinnata, B. fagaroides, Lysiloma acapulcensis, Erythrina spp., Eysendhartia polystachya, Heliocarpus therebinthinaceus, Ceiba aesculifolia, Ipomoea murucoides, Pseudobombax ellipticum y Thevetia peruviana; hay cactáceas de los géneros Pereskia spp., Stenocereus spp., Mammillaria spp.

Cuadro 1. Colecciones científicas que albergan mariposas diurnas recolectadas en Malinalco

\begin{tabular}{clccc}
\hline Siglas & Institución & Registros & Ejemplares & Spp.-sspp. \\
\hline \multirow{2}{*}{ AME } & Allyn Museum of Entomology, Sarasota, FL (hasta 1985) & 55 & 55 & 32 \\
CAS & California Academy of Sciences, San Francisco, CA & 2 & 2 & 2 \\
CNIN & Colección Nacional de Insectos, Instituto de Biología, UNAM & 7 & 7 & 7 \\
MCLB & McGuire Center for Lepidoptera and Biodiversity, Gainesville, FL & 26 & 26 & 16 \\
MZFC & Museo de Zoología Alfonso L. Herrera, Facultad de Ciencias & 9 & 9 & 6 \\
& Ciencias, UNAM & & 1 & 1 \\
USNM & National Museum of Natural History of Washington, DC. & & 100 & 100 \\
& Smithsonian Institution & & 53 \\
\hline
\end{tabular}




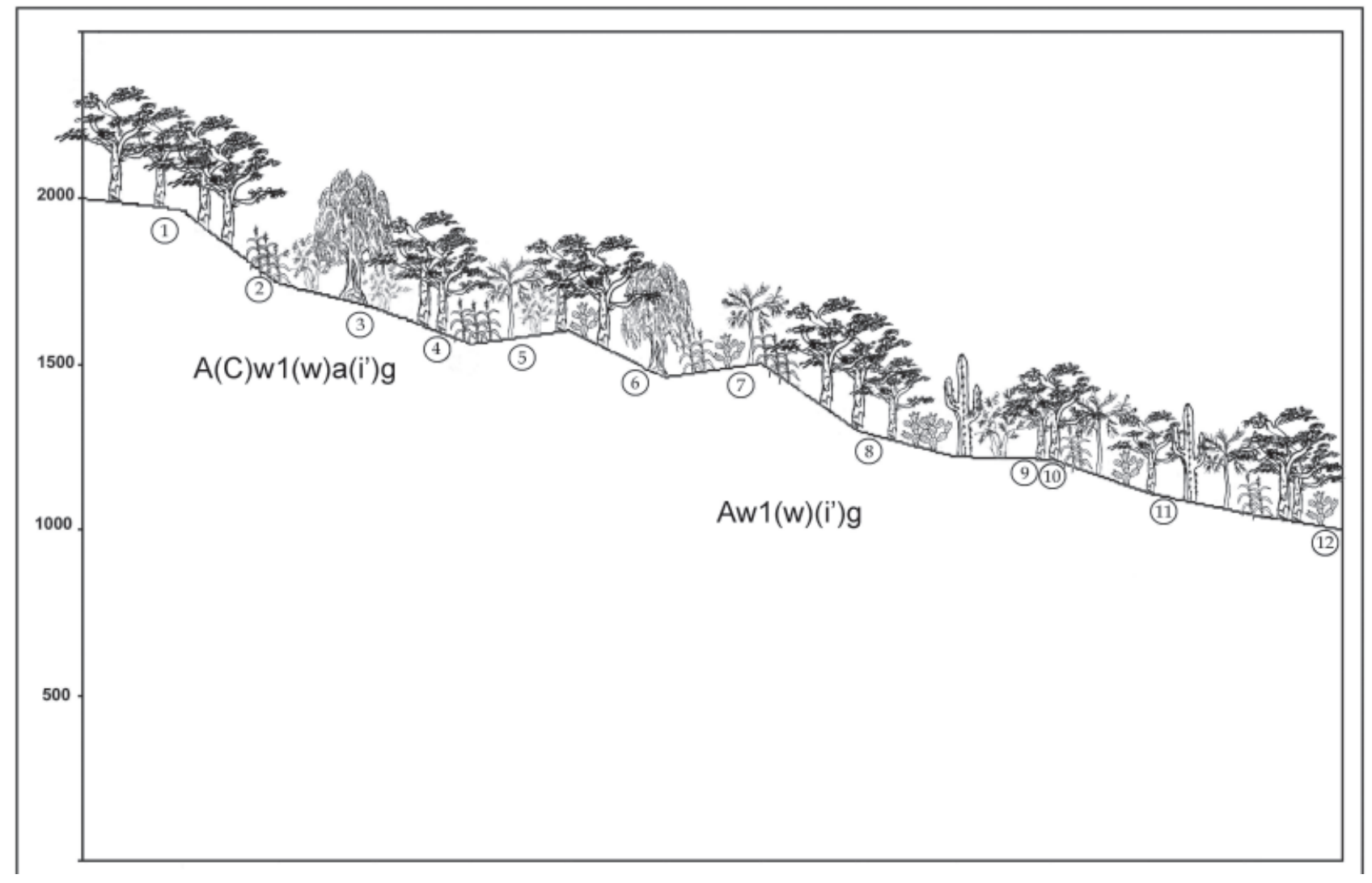

Figura 2. Perfil vegetacional observado en la región de Malinalco.

y Opuntia spp, alternando con la vegetación dominante. En la cima de los cerros se desarrollan zacatonales de Muhlenbergia macroura; en los niveles medios hay elementos de bosque templado (Pinus spp. y Quercus spp.), y en algunas laderas se observa Juniperus flaccida. En lugares con menor elevación, como San Andrés Nicolás Bravo (1 $050 \mathrm{~m}$ ), se observan palmas integradas a la comunidad vegetal principal (Aguilera-Gómez y RivasManzano, 2006). Otro tipo de vegetación, restringido en cuanto a su extensión, es el bosque de galería (BG) que se desarrolla sobre el curso de los ríos y arroyos y tiene como elementos característicos los géneros Taxodium, Salix y Alnus.

En la cabecera municipal y en los ejidos hay plantas ornamentales y huertos de aguacate (Persea americana), anona (Annona muricata), ciruelo (Spondias purpurea), chirimoya (Annona cherimola), cuajinicuil (Inga espuria), guayabo (Psidium guajava), granada (Passiflora edulis), ilama (Annona diversifolia), limón (Citrus limon), mamey (Pouteria sapota), mango (Mangifera indica), nanche (Byrsonima crassifolia), naranjo (Citrus sinensis), níspero
(Eriobotryajaponica), plátano (Musa paradisiaca), toronja (Citrus medica) y zapote blanco (Casimiroa edulis). La agricultura es muy importante en la región, los principales cultivos son de caña de azúcar (Saccharum officinarum), chícharo (Lathyrus sativus), frijol (Phaseolus vulgaris), jitomate (Lycopersicon sculentum), maíz (Zea mays) y tomate (Physalis ixocarpa) (Aguilera-Gómez y RivasManzano, 2006).

Principalmente a causa de la deforestación, la transformación de los hábitats en Malinalco para dedicarlos a la actividad agrícola y ganadera y para la construcción de centros recreativos se ha incrementado en las últimas décadas. Debido a la susceptibilidad de los lepidópteros ante estas modificaciones, poco a poco se han ido desplazando las especies estenoecas, restringiéndose a zonas exclusivas en las cuales aún pueden encontrar los recursos y condiciones necesarias para consumar las etapas de su ciclo de vida. Sin embargo, la escasez de éstos, tanto para la fase larval como para la adulta, repercute en la sobrevivencia poblacional y en el mantenimiento de la diversidad local. 


\section{Materiales y métodos}

Trabajo de campo. En el transcurso de un año se completaron 49 días de recolecta de mariposas diurnas en 12 localidades situadas entre los 1000 y $2000 \mathrm{~m} \mathrm{snm}$ (Fig. 2); las localidades seleccionadas fueron cerro El Zapote ${ }^{1}$, Camino viejo a Ocuilan ${ }^{2}$, El Molino ${ }^{3}$, Jalmolonga ${ }^{4}$, Los Manantiales ${ }^{5}$, Tepehuajes ${ }^{6}$, Puente Caporal ${ }^{7}$, San Pedro Chichicasco $^{8}$, La Angostura9, El Platanar ${ }^{10}$, Colonia Juárez ${ }^{11}$ y San Andrés Nicolás Bravo ${ }^{12}$ (ver Figs. 1 y 2). Una breve descripción de las mismas se presenta en el Cuadro 2.

Las recolectas se iniciaron a las 0900, finalizando a las 1 700; participaron 4 personas por día, aunque se dio el caso de contar con 5 o 6 , incrementándose el esfuerzo de recolecta. Se emplearon 2 técnicas: 1), uso de red entomológica aérea para la búsqueda activa de imagos en los distintos microhábitats: vegetación, caminos o senderos, zonas de penumbra, sitios húmedos, áreas con inflorescencias, y algún otro sitio de afluencia de mariposas y 2), uso de trampas Van Someren-Rydon (Rydon, 1964), que fueron distribuidas en un trayecto de $1 \mathrm{~km}$, colocando una cada $100 \mathrm{~m}, 10$ trampas en total, alternadas lateralmente, tratando de incluir diferentes microhábitats y a una altura de 1.5 a $5 \mathrm{~m}$. Se instalaron en sitios donde no incidieran directamente los rayos solares; el cebo consistió en una mezcla de plátano macho, azúcar mascabado y cerveza, y en algunos casos trozos de naranja y piña. Cada ejemplar fue sacrificado y colocado en una bolsa de papel glassine, rotulada con los datos geográficos de la localidad de recolecta y el sustrato de alimentación.

Determinación taxonómica. Se realizó en el Departamento de Zoología del Instituto de Biología (IBUNAM). Para ello se preparó una muestra representativa de las especies recolectadas con base en las técnicas sugeridas por Morón y Terrón (1988). La determinación se llevó a cabo mediante comparación con los ejemplares depositados en la Colección Nacional de Insectos, sección Lepidoptera (IBUNAM), así como en la Colección de Lepidópteros del Museo de Zoología Alfonso L. Herrera de la Facultad de Ciencias, UNAM (MZFC), y mediante la revisión de las obras de Godman y Salvin (1869 - 1901), Seitz (1907 1924), Howe (1975), Scott (1986), De la Maza (1987), De Vries (1987a,b) Luis-Martínez et al. (2003) y LlorenteBousquets et al. (1997). En el caso de Hesperioidea se requirió confirmar la determinación mediante el uso de claves (Evans 1951, 1952, 1953, 1955) y otros textos, bajo el examen de los genitales. Cada ejemplar se rotuló con la información taxonómica y de campo correspondiente. Cerca del $85 \%$ de los ejemplares se depositó en la Colección Nacional de Insectos (IBUNAM) y un 15\% en el Museo Universitario Dr. Luis Mario Schneider Z. ubicado en Malinalco.

Manejo de la Información. La información obtenida en campo se transcribió a una base de datos diseñada en el programa Excel 97; esta base incluyó datos de recolecta y taxonómicos, refiriendo para cada ejemplar la siguiente información: número de ejemplar, nombre científico, localidad de recolecta, ubicación geográfica, altitud, tipo de vegetación, fecha y sustrato de alimentación. La base creada proporcionó la información necesaria y el manejo expedito de los datos para realizar los análisis correspondientes y desarrollar los objetivos planteados.

\section{Resultados}

Curva de acumulación de especies. Con base en los datos de recolecta y bibliográficos se integró una lista general de 240 especies de mariposas diurnas para Malinalco. Al emplear el modelo de Clench (1979), se observó que el número teórico de especies esperado puede aproximarse a 265, es decir, se esperaría encontrar 25 especies más en la región. Cabe mencionar que en las últimas 2 recolectas no se adicionaron nuevas especies a la lista. No obstante, quizá sea adecuado elegir otras localidades intermedias y aumentar los días de campo hasta disminuir los nuevos registros.

Con base en la curva elaborada se recolectó el $81 \%$ de las especies, lo cual muestra la representatividad de este trabajo (Fig. 3).

La determinación taxonómica de los 1333 imagos recolectados permitió obtener la lista de las mariposas diurnas presentes en Malinalco integrada por 2 superfamilias, 6 familias, 21 subfamilias, 147 géneros y 213 especies (Apéndice 1, Cuadro 3). Se dan a conocer 157 nuevos registros (63 regionales y 94 estatales). Los géneros Urbanus, Strymon, Emesis y Chlosyne fueron los más diversos, al considerar aquellos con más de 4 especies.

A la lista generada con el trabajo de campo y colecciones científicas, se adicionaron 27 especies citadas en la literatura para la zona, no recolectadas en este proyecto, registrando un total de 240 especies para Malinalco. La lista integral que se presenta en el Apéndice 1 sigue un arreglo filogenético aproximado, con base en la nomenclatura propuesta por Warren (2000) y LlorenteBousquets et al. (2006) para Hesperioidea y Papilionoidea, respectivamente.

La superfamilia Hesperioidea, con una familia (Hesperiidae), incluyó 4 subfamilias, 58 géneros y 81 especies y la Papilionoidea con 5 familias, incluyó 17 subfamilias, 99 géneros y 159 especies, cifras que representan el $85 \%$ y el $69 \%$ de las especies de 
Cuadro 2. Características geográfico-ecológicas de las localidades de recolecta

\begin{tabular}{|c|c|c|}
\hline Localidad & $\mathrm{Veg}$ & Descripción \\
\hline $\begin{array}{l}\text { Cerro El Zapote } \\
18^{\circ} 57^{\prime}, 09 " \mathrm{~N} \\
99^{\circ} 30^{\prime} 41^{\prime \prime} \mathrm{O}\end{array}$ & $\mathrm{SBC}$ & $\begin{array}{l}\text { En la cima se encuentra la SBC conservada; al descender, en el punto más bajo } \\
\text { de la ladera la vegetación ha sido desplazada por potreros y establos. }\end{array}$ \\
\hline
\end{tabular}

$1964 \mathrm{~m}$

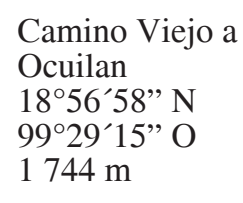

El Molino

$18^{\circ} 55^{\prime} 28^{\prime \prime} \mathrm{N}$

$99^{\circ} 29^{\prime} 56^{\prime \prime} \mathrm{O}$

$1670 \mathrm{~m}$

$\mathrm{Vs}$

Zc

$\mathrm{Zc}$

Vs

$\mathrm{Zc}$

Los Manantiales

$18^{\circ} 55^{\prime} 36^{\prime \prime} \mathrm{N}$

$99^{\circ} 26^{\prime} 14$ " O

$1601 \mathrm{~m}$

Jalmolonga

$18^{\circ} 55^{\prime} 01$ " N

$99^{\circ} 29^{\prime} 04^{\prime \prime} \mathrm{O}$

$1560 \mathrm{~m}$

Puente Caporal

$18^{\circ} 53$ '59" N

$99^{\circ} 27^{\prime} 32^{\prime \prime} \mathrm{O}$

$1503 \mathrm{~m}$

Tepehuajes

$18^{\circ} 53^{\prime} 01$ ' N

$99^{\circ} 27^{\prime} 43^{\prime \prime} \mathrm{O}$

$1462 \mathrm{~m}$

San Pedro

Chichicasco

$18^{\circ} 50$ ' 34 " N

$99^{\circ} 27^{\prime} 38^{\prime \prime} \mathrm{O}$

$1297 \mathrm{~m}$

La Angostura

$18^{\circ} 47^{\prime} 25^{\prime \prime} \mathrm{N}$

$99^{\circ} 27^{\prime} 29^{\prime \prime} \mathrm{O}$

$1221 \mathrm{~m}$

El Platanar

$18^{\circ} 53^{\prime} 57^{\prime \prime} \mathrm{N}$

$99^{\circ} 27^{\prime} 41^{\prime \prime O}$

$1214 \mathrm{~m}$

SBC

BG

$\mathrm{Zc}$ Vs

Vs

$\mathrm{Vr}$

$\mathrm{Zc}$

SBC

BG

$\mathrm{Zc}$

Zc
Área abierta, empleada para el cultivo de maíz y el establecimiento de potreros.

Área abierta, altamente perturbada por el establecimiento de estanques piscícolas, el comercio y la siembra de maíz, caña de azúcar y gladiolo; existen viveros de ornamentales y frutales. Se recolectó sobre el margen del río cuyo cauce va acompañado por vegetación riparia y algunos árboles de Salix babilonica.

SBC Área muy perturbada por el establecimiento de centros recreativos, sólo

Area muy perturbada por el establecimiento de centros recreativos, sólo ornamentales y frutales. El río Chalma cruza esta zona y se encuentra contaminado principalmente por desechos inorgánicos.

Área plana, perturbada por la siembra de maíz y gladiolo. Existe una pequeña laguna artificial que en su límite alberga vegetación riparia.

Área de cultivo, los principales son: maíz, gladiolo y caña de azúcar. Se recolectó en el margen de la carretera y a orilla del río; conforme se introduce a la zona siguiendo el cause se encuentran elementos de la SBC y BG, representado por Salix babilonica, Taxodium sp. y Alnus sp., alternando con guayabos.

La vegetación original ha sido sustituida por el cultivo de maíz, caña de azúcar, trigo, gladiolo y el establecimiento de potreros.

Esta zona alberga la presa hidroeléctrica Alameda, es una cañada cuyas laderas difieren en cuanto al tipo de vegetación, una de ellas aún presenta áreas donde se puede encontrar SBC conservada, en contraste la otra presenta asentamientos humanos y extensos huertos de ciruelo, mango y cultivos de maíz.

Cañada con pendientes abruptas, una de ellas se utiliza para el cultivo de maíz y gladiolo, así como amplios potreros donde se recolectaron especies acimófagas; en contraste, la otra ladera esta muy conservada y los elementos de la SBC alternan con cactus candelabriformes del género Pereskia, Stenocereus y Mammilaria.

Cañada pequeña que desemboca al río. En esta localidad una de las laderas aún presenta áreas conservadas de SBC, sin embargo, en áreas de menor pendiente se han establecido potreros; en la otra ladera existe asentamiento humano, huertos de plátano, mango, mamey y algunos cítricos; ambas cañadas se encuentran separadas por el río de aproximadamente $10 \mathrm{~m}$ de ancho. En esta localidad existe BG restringido al margen del río, se recolectó en una playita que se forma al descender el nivel del agua. 
Cuadro 2. Continúa

\begin{tabular}{|c|c|c|}
\hline Localidad & Veg & Descripción \\
\hline $\begin{array}{l}\text { Colonia Juárez } \\
18^{\circ} 47^{\prime} 58^{\prime \prime} \mathrm{N} \\
99^{\circ} 27^{\prime} 18^{\prime \prime} \mathrm{O} \\
1111 \mathrm{~m}\end{array}$ & $\begin{array}{l}\text { SBC } \\
\text { Vs } \\
\mathrm{Zc}\end{array}$ & $\begin{array}{l}\text { Cañada con pendientes abruptas que desembocan al río, una de ellas aún } \\
\text { presenta SBC conservada y alterna con cactus candelabriformes del género } \\
\text { Pereskia y Stenocereus. La otra ladera se emplea para el cultivo principalmente } \\
\text { de maíz y gladiolo, existen extensos huertos de ciruelo, anona, chichozapote, } \\
\text { plátano, mamey, mango y aguacate; en zonas con menor pendiente se han } \\
\text { conservado elementos de la vegetación original que sirven de protección a } \\
\text { los cultivos de café (Coffea arabica), lo que genera que existan áreas con } \\
\text { penumbra. Ocasionalmente se recorrió el poblado, donde hay extensas bugam- } \\
\text { bilias. }\end{array}$ \\
\hline $\begin{array}{l}\text { San Andrés Nicolás } \\
\text { Bravo } \\
18^{\circ} 46^{\prime} 12^{\prime}, \mathrm{N} \\
99^{\circ} 27^{\prime} 20^{\prime} \mathrm{O} \\
1050 \mathrm{~m}\end{array}$ & $\begin{array}{l}\text { SBC } \\
\text { Pal } \\
\text { Vs }\end{array}$ & $\begin{array}{l}\text { Es la zona con menor altitud, limita con el estado de Morelos, ambas laderas } \\
\text { se encuentran semiconservadas en cuanto al tipo de vegetación. Existen } \\
\text { huertos de mango, plátano, mamey y papayos; en los lugares más planos se } \\
\text { ha sembrado maíz y café. En el poblado hay diversas ornamentales donde } \\
\text { generalmente se recolectaron piéridas y papiliónidas. }\end{array}$ \\
\hline
\end{tabular}

Tipos de vegetación: $\mathrm{BG}=$ bosque de galería, Pal=Palmar, $\mathrm{SBC}=$ selva baja caducifolia; $\mathrm{Vr}=$ vegetación riparia, Vs=vegetación secundaria y Zc=zona de cultivo.

Modelo de Clench: $\mathrm{y}=\left((264.4518)^{*} \mathrm{x}\right) /((11.59243)+\mathrm{x})$

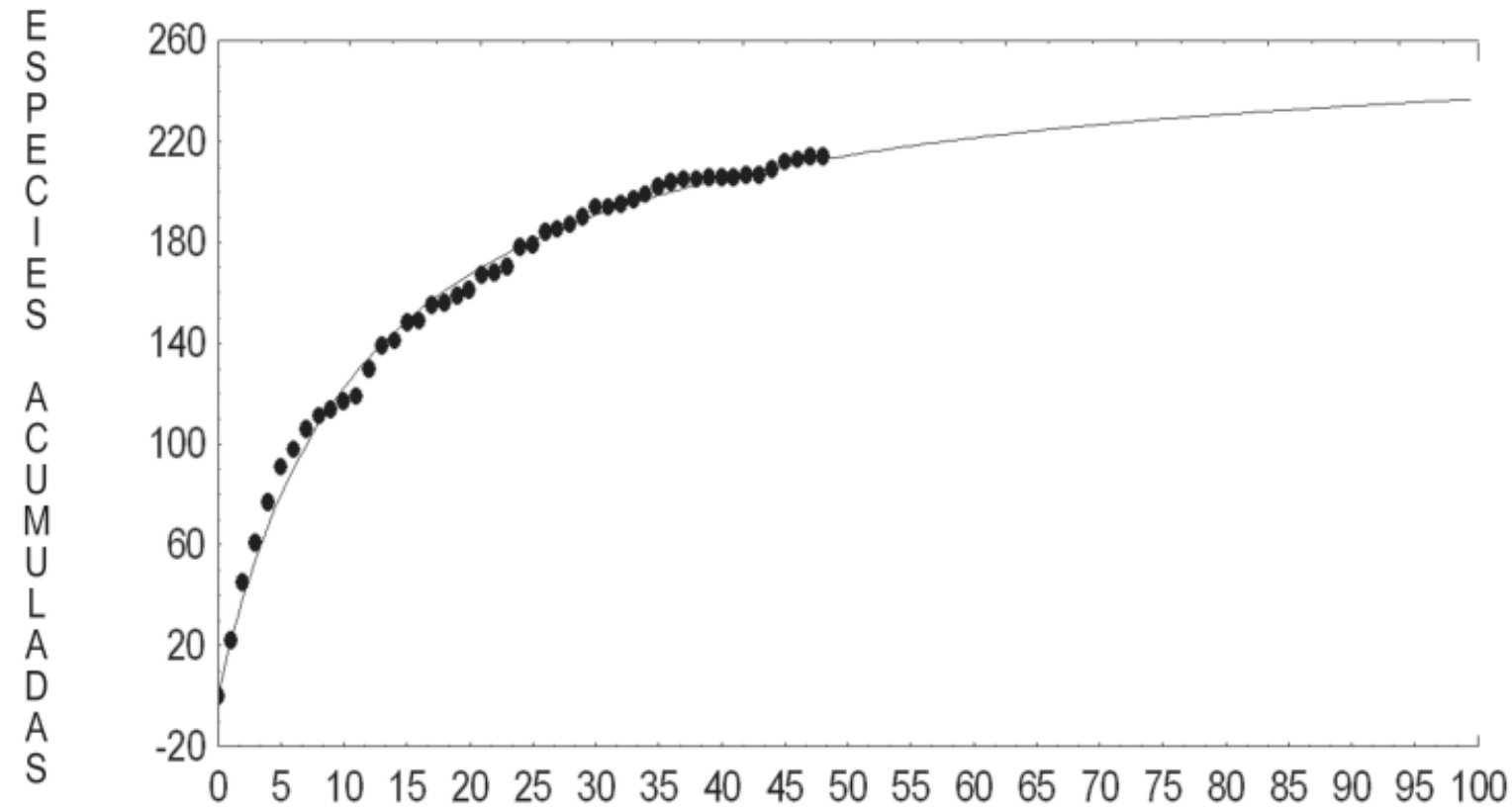

\section{RECOLECTAS}

Figura 3. Curva de acumulación de especies empleando el modelo de Clench (1979). 
Cuadro 3. Composición faunística de las superfamilias Hesperioidea* y Papilionoidea recolectadas en Malinalco durante 2001-2002

\begin{tabular}{cccc}
\hline Familia & Subfamilia & Género & $\begin{array}{c}\text { Especies- } \\
\text { Subespecies }\end{array}$ \\
\hline Hesperiidae* & 4 & 56 & 74 \\
Papilionidae & 2 & 8 & 16 \\
Pieridae & 3 & 16 & 26 \\
Lycaenidae & 2 & 21 & 30 \\
Riodinidae & 1 & 8 & 12 \\
Nymphalidae & 9 & 38 & 55 \\
Total & 21 & 147 & 213 \\
\hline
\end{tabular}

hesperioideas y papilionoideas registradas para el Estado de México (Cuadro 4).

La familia Hesperiidae presentó la mayor riqueza específica en Malinalco con 74 especies (35\% del total) en 56 géneros. Warren (2000) mencionó que las hespéridas consistentemente constituyen cerca del $40 \%$ de las mariposas diurnas presentes en cualquier localidad en la República Mexicana, sin importar la época o el ambiente donde se recolecten. Llorente-Bousquets et al. (1990) estimaron que existen cerca de 800 especies en el país, pero actualmente se conocen 741 , cifra que simboliza casi el $40 \%$ de la lepidopterofauna diurna de México. Con base en el análisis de los datos de recolecta y la revisión de la literatura, se registraron algunas hespéridas como nuevos registros (61 regionales y 52 estatales).

Para la familia Papilionidae se registraron 8 géneros (el más diverso es Parides con 3 especies: P. erithalion trichopus, $P$. montezuma y $P$. photinus) y 16 especies. Se recolectaron hembras polimorfas de Baronia b. brevicornis, mariposa endémica de México y nuevo registro estatal, procedentes de las localidades de El Platanar (1214 m), San Pedro Chichicasco (1297 m) y La Angostura (1221 m), zonas donde predomina Acacia cochliacantha, planta de alimentación para la fase larval. El imago aparece en un periodo muy corto, al final de mayo y principio de junio, hecho relacionado con el inicio de la temporada de lluvia (Vázquez y Pérez, 1962).

La familia Pieridae (16 géneros y 26 especies) incluye especies multivoltinas y euriecas, como Phoebis sennae marcellina, Pyrisitia dina weswoodi, P. proterpia, Eurema daira sidonia, Nathalis iole y Leptophobia aripa elodia, que son frecuentes en varias localidades de recolecta y vuelan la mayor parte del año, hecho posiblemente asociado a 3
Cuadro 4. Ropalocerofauna registrada para el Estado de México y Malinalco

\begin{tabular}{lrrc}
\hline Familia & MEX & Malinalco & \multicolumn{1}{l}{$\%$} \\
\hline Hesperiidae & 95 & 74 & 77.8 \\
Papilionidae & 19 & 16 & 84.2 \\
Pieridae & 35 & 26 & 74.2 \\
Lycaenidae & 54 & 30 & 55.5 \\
Riodinidae & 20 & 12 & 60 \\
Nymphalidae & 102 & 55 & 53.9 \\
Total & 325 & 213 & 65.5 \\
\hline
\end{tabular}

$M E X=E s t a d o$ de México, $(\%)$ porcentaje de las especies presentes en Malinalco respecto al registro estatal.

factores: 1), una valencia ecológica amplia; 2), ausencia de competidores y 3), capacidad de explotar mayor variedad de recursos, en comparación con Enantia mazai diazi, Rhabdodryas trite, Eurema arbela boisduvaliana y Pieriballia v. viardi, especies exclusivas de una localidad y presentes sólo algunos meses.

La familia Lycaenidae se integró con 21 géneros (el más diverso es Strymon) y 30 especies, algunas muy abundantes como Cupido comyntas, Leptotes marina, Hemiargus hanno antibubastus y Echinargus isola. No obstante, la mayoría de las licénidas mostró una densidad poblacional muy baja, lo cual es característico de algunas especies tropicales (Lamas, 1981), entre ellas se menciona Brangas neora, Ocaria ocrisia, Cyanophrys herodotus, Ziegleria guzanta, Calycopis clarina, Aubergina paetus y Chalybs hassan, de las cuales se recolectaron muy pocos ejemplares.

El $75 \%$ de las especies de riodínidos son de afinidad neotropical y el $25 \%$ neártica; entre ellas se encuentra Emesis z. zela, recolectada exclusivamente en el cerro El Zapote (1 964 m snm). En Riodinidae, Emesis y Calephelis son los géneros más diversos. Calephelis sixola es multivoltina y frecuente en los 12 sitios de recolecta, a diferencia de Rhetus arcius beutelspacheri, Baeotis z. zonata y Apodemia walkeri que se pueden considerar univoltinas, con densidades poblacionales muy bajas (se cuenta con 1 o 2 ejemplares), e inclusive pueden ser especies raras en la zona.

La familia Nymphalidae incluyó 38 géneros y 55 especies. Los géneros más diversos son Chlosyne y Anthanassa. Las especies frecuentes y multivoltinas fueron Morpho p. polyphemus, Hermeuptychia hermes, Anartia f. fatima, Chlosyne ehrenbergii, Anthanassa nebulosa alexon, 
A. t. texana y Heliconius charithonia vazquezae, a diferencia de Manataria hercyna maculata, Cyllopsis hilaria, Biblis hyperia aganisa, Temenis laothoe quilapayunia, Chlosyne j. janais, Texola anomalus coracara, Anthanassa frisia tulcis y Euptoieta claudia daunius, que se recolectaron en una localidad y en determinada época del año.

\section{Discusión}

Gremios alimentarios. Con base en el tipo de sustrato de alimentación, Vargas-Fernández et al. (1992) clasificaron las preferencias de las mariposas diurnas en 3 gremios: 1), nectarívoras $(\mathrm{N})$, que obtienen recursos a partir del néctar de las flores; 2), hidrófilas $(\mathrm{H})$, que toman sus nutrimentos principalmente en la arena húmeda o charcos y 3), acimófagas (A), que llegan a frutos en estado de fermentación, excretas de algunos vertebrados (principalmente aves y mamíferos), carroña u otro tipo de materia orgánica en descomposición. No obstante, hay especies que pueden pertenecer a más de un gremio, dependiendo de la zona geográfica que habitan y las condiciones ambientales (Young, 1975). Es complejo asignar a una especie dentro de uno o más gremios al considerar que existen diferentes variables que influyen en su conducta y preferencia alimentaria, entre ellas la abundancia y disponibilidad del recurso -según sea la época del año-, los requerimientos nutricionales para cada sexo, y la capacidad que presentan las especies para acudir a uno o más sustratos. En este estudio se consideran los 3 gremios en Malinalco.

En el área de estudio, las mariposas que se alimentan de néctar constituyeron el $54 \%$ de las especies recolectadas, lo cual muestra la importancia de las flores como principal recurso para la obtención de carbohidratos y agua (Murphy et al., 1983; Karlsson, 1994, 1995). Al considerar un gremio $(\mathrm{N})$ y 3 subgremios generalistas $(\mathrm{N}+\mathrm{H}),(\mathrm{N}+\mathrm{A})$ y $(\mathrm{N}+\mathrm{H}+\mathrm{A})$ se comprende el $93 \%$ del total (213 especies). Las hidrófilas $(\mathrm{H})$, que frecuentemente visitaban los charcos o zonas húmedas en busca de agua y minerales, representaron el 1\% (3). Las acimófagas (A), es decir, aquellas especies que fueron observadas alimentándose exclusivamente de materia orgánica en descomposición registraron $4 \%$ (8). Al considerar un gremio y 3 subgremios, Gremio $(\mathrm{H})=(\mathrm{N}+\mathrm{H}),(\mathrm{H}+\mathrm{A})$ y $(\mathrm{N}+\mathrm{H}+\mathrm{A})$ y Gremio $(\mathrm{A})=$ $(\mathrm{N}+\mathrm{A}),(\mathrm{H}+\mathrm{A})$ y $(\mathrm{N}+\mathrm{H}+\mathrm{A})$, potencialmente las hidrófilas y acimófagas comprenden el $40 \%$ y $21 \%$ de las especies, respectivamente (Cuadro 5).

En Hesperiidae, el $93 \%$ de las especies son nectarívoras, el $4 \%$ se incluye en el subgremio $(\mathrm{N}+\mathrm{A})$, y sólo una especie, Pyrgus oileus, explota los 3 recursos $(\mathrm{N}+\mathrm{H}+\mathrm{A})$. Generalmente los géneros Urbanus, Chioides y Aguna se observaron alimentándose en Bouganvillea glabra y Astraptes en excremento de ave.

En Papilionidae, el subgremio $(\mathrm{N}+\mathrm{H})$ es el mejor representado (69\%), seguido por los nectarívoros y los que explotan diversos sustratos $(\mathrm{N}+\mathrm{H}+\mathrm{A})$, a este último pertenecen las especies Parides montezuma y Parides photinus.

En Pieridae, el primer lugar lo ocupó el gremio nectarívoros $(\mathrm{N})(50 \%)$; le siguen aquellas especies que recurren a más de un sustrato. Las especies Phoebis $p$. philea, Pyrisitia dina westwoodi, P. proterpia, Eurema daira sidonia y E. m. mexicana, del subgremio ( $\mathrm{N}+\mathrm{H}+\mathrm{A})$, con frecuencia se observaron alimentándose en flores e inflorescencias de leguminosas, frutos en descomposición (chicozapote, mango y guanábana), formando pequeños grupos en estiércol de vaca o caballo, y en charcos a los

Cuadro 5. Gremios y subgremios alimentarios por familia

\begin{tabular}{|c|c|c|c|c|c|c|c|c|}
\hline Gremio & Hesperiidae & Papilionidae & Pieridae & Lycaenidae & Riodinidae & Nymphalidae & Total & $\%$ \\
\hline $\mathrm{N}$ & 69 & 3 & 13 & 15 & 4 & 7 & 111 & 52 \\
\hline $\mathrm{H}$ & 0 & 0 & 0 & 0 & 1 & 2 & 3 & 1 \\
\hline A & 0 & 0 & 0 & 0 & 0 & 8 & 8 & 4 \\
\hline $\mathrm{N}+\mathrm{H}$ & 1 & 11 & 6 & 9 & 4 & 23 & 54 & 25 \\
\hline $\mathrm{N}+\mathrm{A}$ & 3 & 0 & 1 & 0 & 0 & 2 & 6 & 3 \\
\hline $\mathrm{H}+\mathrm{A}$ & 0 & 0 & 0 & 0 & 1 & 3 & 4 & 1 \\
\hline $\mathrm{N}+\mathrm{H}+\mathrm{A}$ & 1 & 2 & 6 & 6 & 2 & 10 & 27 & 13 \\
\hline Especies & 74 & 16 & 26 & 30 & 12 & 54 & 213 & 100 \\
\hline
\end{tabular}

Gremios: $\mathrm{N}=$ nectarívoro, $\mathrm{H}=$ hidrófilo y $\mathrm{A}=$ acimófago.

Subgremios $=(\mathrm{N}+\mathrm{H}),(\mathrm{N}+\mathrm{A}),(\mathrm{H}+\mathrm{A}),(\mathrm{N}+\mathrm{H}+\mathrm{A})$ 
que probablemente acudían con el fin de obtener agua, detritos y sales (Rydon, 1964; Downes, 1973; Arms et al., 1974; Sevastopulo, 1974).

En Lycaenidae, todas las especies son potencialmente nectarívoras si se considera un gremio (N) y 2 subgremios $(\mathrm{N}+\mathrm{H}$ y $\mathrm{N}+\mathrm{H}+\mathrm{A})$. El $20 \%$ puede utilizar gran diversidad de recursos e incluirse en $(\mathrm{N}+\mathrm{H}+\mathrm{A})$, tal es el caso de Leptotes marina y Echinargus isola, que se observaron alimentándose sobre estiércol de vaca y caballo, animales muertos (aves, mamíferos pequeños y reptiles) y frutos en descomposición (chicozapote, guanábana, guayaba, mango y plátano); posiblemente esta versatilidad en la elección del alimento (eurifagia) influye para que ambas especies sean comunes en las 12 localidades de recolecta y vuelen la mayor parte del año (multivoltinas). Se observó que las flores de leguminosas (Acacia spp., Lysiloma spp., Phaseolus spp. y Prosopis spp.) son las más visitadas por las licénidas.

En Riodinidae, potencialmente el $83 \%$ de las especies son nectarívoras $(\mathrm{N})$, el $67 \%$ hidrófilas $(\mathrm{H})$ y el $17 \%$ acimófagas (A); en este gremio se encuentra Rhetus arcius beutelspacheri, especie recolectada sobre estiércol de caballo, el cual posiblemente aporta aminoácidos y algunos componentes nitrogenados.

La familia Nymphalidae presenta la mayor variedad de hábitos alimentarios, pues es la única en que todos los gremios y subgremios se representan; se considera que las especies son eurífagas, es decir, son capaces de aprovechar uno o más sustratos para alimentarse. Sin embargo, el valor más alto es el subgremio nectarívoroshidrófilos $(\mathrm{N}+\mathrm{H})$ con el $42 \%$ de las especies; no obstante, el $41 \%$ de las especies son potencialmente acimófagas (A). Las acimófagas incluyen las que fueron recolectadas mediante la trampa Van Someren-Rydon, como Myscelia c. cyananthe (exclusivamente hembras), Taygetis weymeri, Anaea troglodyta aidea y Temenis laothoe quilapayunia, $\mathrm{u}$ observadas en materia orgánica en descomposición, tal es el caso de Morpho p. polyphemus (en mango y naranja), Cissia similis, C. themis y Hermeuptychia hermes (en chicozapote, mangos y guanábanas maduras), Siproeta stelenes biplagiata (en guayabos y excremento de ave) y Archaeoprepona demophon occidentalis (en chicozapote, mamey y excremento de mamífero).

Distribución altitudinal. En Malinalco se presenta la selva baja caducifolia la cual, de acuerdo con Miranda y Hernández-Xolocotzi (1963), se desarrolla en un intervalo de 0 a $1900 \mathrm{~m}$ snm. Griffiths (1985) mencionó que la variación de la altitud ejerce una acción importante sobre el clima, principalmente en la disminución o el aumento de la presión atmosférica, el cambio en la densidad del aire, el descenso de la temperatura y el aumento de la precipitación. La información geográfico-climática disponible para el área no nos permite efectuar un análisis como el que sugiere Griffiths.

Los datos de recolecta muestran que existe un decremento en la riqueza de mariposas conforme la altitud se incrementa. El $31 \%$ de las especies (65) establece sus poblaciones por debajo de los $1400 \mathrm{~m}$, en donde las hespéridas son las más abundantes con el $46 \%$ (30); en cambio, algunos componentes faunísticos tienen distribución amplia en los 5 pisos altitudinales propuestos (Fig. 4). Un estudio que implique correlación estadística requiere muestreos más detallados.

De las 213 especies estudiadas en Malinalco, 24 (11\%) se distribuyen en las 12 localidades, 7 en 6,2 en 5,32 en 3 o 4 y 47 en 2 (Cuadro 2 para referencia), mientras 60 (28\%) resultaron exclusivas de alguna localidad; Puente Caporal (1503 m snm) es la localidad que presentó el mayor número de especies exclusivas (11): Enantia mazai diazi, Ocaria ocrisia, Strymon astiocha, Baeotis z. zonata, Epiphile adrasta escalantei, Nymphalis a. antiopa, Spathilepia clonius, Nisoniades ephora, Eantis tamenund, Wallengrenia o. otho y Panoquina o. ocola, es importante mencionar que en estos casos no se incluyen datos bibliográficos.

Las localidades más diversas son Colonia Juárez (1111 m snm) con 108 especies y 190 ejemplares, cifras que representan el $51 \%$ de las especies y el $14 \%$ de los individuos recolectados, y El Platanar (1 $214 \mathrm{~m}$ ) con 101 especies (47\%). Ambas localidades cuentan con el mayor número de días de recolecta como resultado de mostrar, en el transcurso del año, condiciones ambientales óptimas y disponibilidad de recursos alimenticios (flores, frutos, materia orgánica, zonas húmedas, etc.) para los imagos.

La localidad de menor diversidad es Los Manantiales (1601 m snm) con 7 especies (3\% del total), sin registros para las familias Papilionidae y Pieridae; en estos resultados

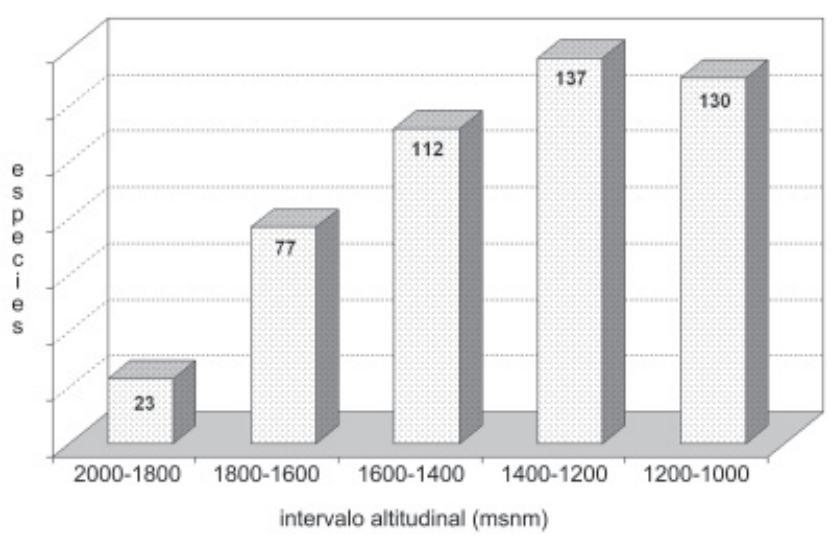

Figura 4. Riqueza de especies de hesperioideas y papilionoideas dentro de un intervalo altitudinal. 
influyeron las características de la zona, el difícil acceso, el alto grado de perturbación que presenta y el esfuerzo de recolecta limitado.

El cerro El Zapote (1964 m), el punto más alto para la SBC, se encuentra conservado favoreciendo la existencia de amplias zonas de penumbra; no obstante, se hallaron algunos claros formados por la caída de árboles, donde se recolectaron imagos que comúnmente se alimentaban en flores. Esta localidad incluyó al mayor número de registros para Euptychia fetna y con frecuencia se observó el vuelo diurno de los satúrnidos Paradirphia fumosa y $P$. michoacana; la familia mejor representada fue Hesperiidae con 7 especies.

San Andrés Nicolás Bravo es la localidad de menor altitud (1050 m), limita al sur con el estado de Morelos, de diciembre a abril es extremamente seco, el recurso es escaso y restringido a la orilla del río y a las zonas de cultivo; sólo se registraron 71 especies; 6 son exclusivas de la zona.

Distribución altitudinal por familia. Las familias Papilionidae, Pieridae, Riodinidae y Nymphalidae poseen alta riqueza de especies en la Colonia Juárez (1 111 m), Hesperiidae en El Platanar (1 214 m) y Lycaenidae en El
Molino (1 670 m). Como se observa, 5 de las 6 familias consideradas tienen amplia representación de especies en el intervalo altitudinal inferior a los 1400 m (Fig. 5).

Hesperiidae presentó el mayor número de especies exclusivas (8) en El Platanar, localidad donde cuenta con el mayor número de especies (32); los ejemplares se recolectaron en áreas de vegetación riparia. Los géneros Chalypyge sp., Apyrrothrix sp., Aguna spp. y Astraptes spp., se hallaron volando en cultivos de café que se encuentran protegidos por árboles de mango de aproximadamente $15 \mathrm{~m}$ de altura, lo que genera un microhábitat húmedo y sombrío, propio de estos géneros.

La familia Pieridae exhibió su mayor riqueza (17 especies) en el Platanar (1 $214 \mathrm{~m}$ ) y en Colonia Juárez $(1111 \mathrm{~m})$, en esta última se tiene el registro de Pieriballia v. viardi, con un solo ejemplar recolectado durante el tiempo que duró la investigación, lo cual sugiere que sea una especie ocasional.

La familia Riodinidae registró el $67 \%$ de las especies en localidades menores a $1250 \mathrm{~m}$; frecuentemente fueron observadas en el sotobosque, o posando sobre las hojas con las alas extendidas en sitios abiertos donde abundaban flores o pequeños charcos.

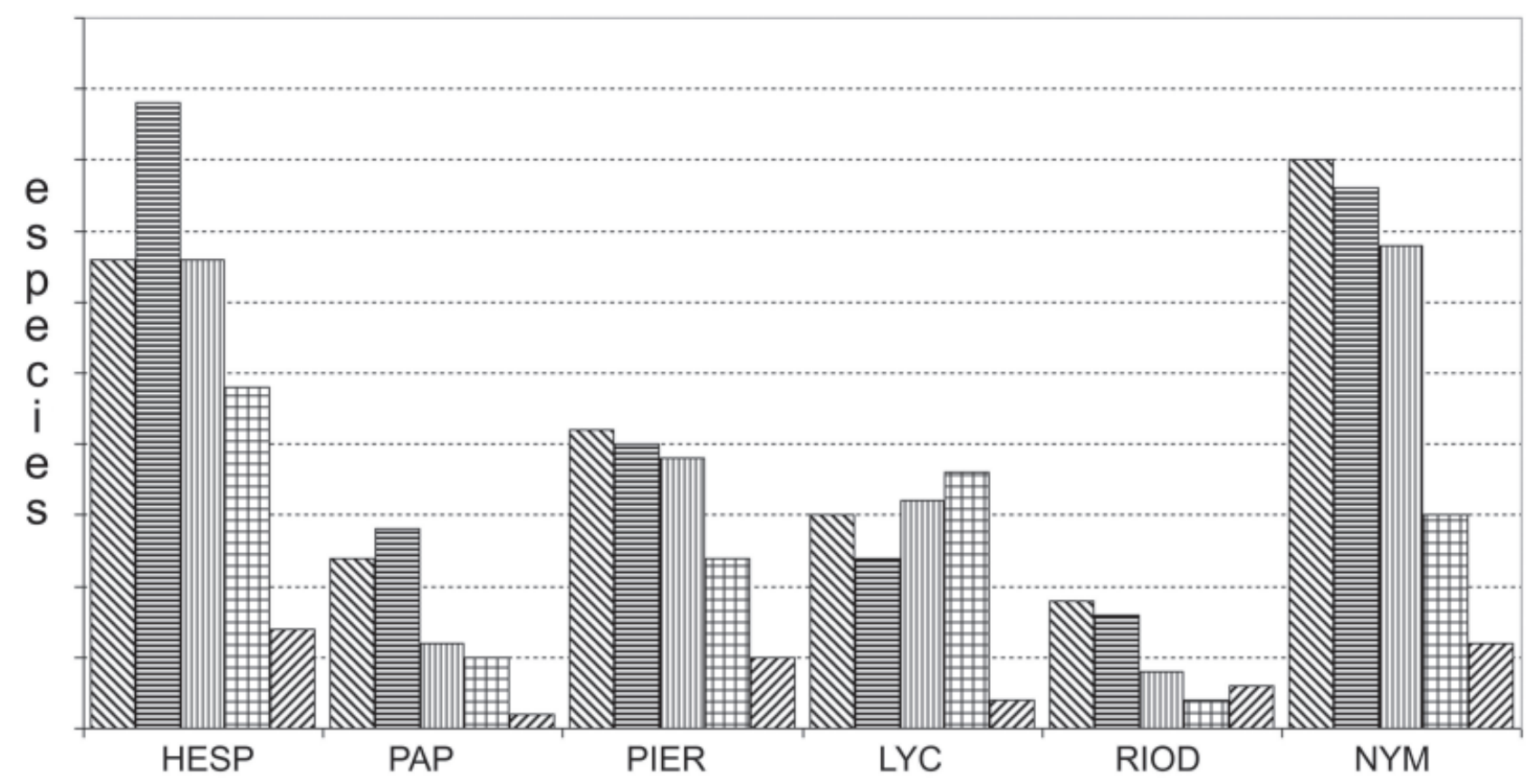

$\begin{array}{lllllll}N 1000-1200 & \equiv 1200-1400 \quad \text { || } 1400-1600 & \text { ष } 1600-1800 \text { Z } 1800-2000\end{array}$

\section{intervalo altitudinal (msnm)}

Figura 5. Distribución altitudinal por familias en el intervalo altitudinal 1000 - 2000 msnm. 
De las 31 especies de Lycaenidae registradas, el 55\% (17) fue recolectado en El Molino (1 670 m), localidad exclusiva para Cyanophrys herodotus, Calycopis clarina y Tmolus echion; esto puede deberse a que es un área abierta, altamente perturbada, en donde abundan arvenses, ruderales y pequeñas leguminosas de las que se alimentan, así como zonas húmedas y potreros donde se concentran en grupos pequeños. Cabe mencionar que en comunidades alteradas, numerosas herbáceas que florecen durante largos periodos, los cuales se traslapan, se desarrollan continuamente y compiten por los polinizadores disponibles, pues se exhiben como un recurso atractivo para los imagos.

Estacionalidad de las Hesperioidea y Papilionoidea. En Malinalco el periodo de sequía tiene una duración de aproximadamente 7 meses, desde finales de noviembre hasta mayo, siendo diciembre el mes más seco. En esta época se presenta el punto máximo de florecimiento y fructificación de los árboles en la selva baja caducifolia (Miranda y Hernández-Xolocotzi, 1963). Janzen (1967) señaló que el florecimiento en la estación seca conlleva numerosas ventajas, los insectos polinizadores (heliófilos) resultan favorecidos por las condiciones soleadas; la falta de hojas hace que las flores y frutos sean más visibles para ellos, mejorando su actividad durante la estación. En la zona de estudio existe disponibilidad de recursos alimenticios en este periodo, principalmente inflorescencias de diversas especies, y frutos de anona, ciruelo, chicozapote, mamey, mango, guayabo, granada y plátano, todos ellos introducidos, aunque algunos desde hace mucho tiempo.

García (1988) indicó una precipitación promedio de $5.5 \mathrm{~mm}$ en el mes de mayo para la zona de Malinalco. De acuerdo con nuestras observaciones, hacia finales de este mes se iniciaron las primeras lluvias y se tuvo la mayor riqueza, registrándose el vuelo de 102 especies (48\%), a diferencia del mes de enero, cuando sólo fueron 20 (9\%).

El periodo de lluvias comprende 4 meses (junio a septiembre); en este lapso es notable el cambio en la fisonomía de la vegetación debido al brote de las plantas herbáceas anuales y al desarrollo foliar de las perennes, que principalmente sirven de alimento a la fase larval

\section{$\operatorname{Pp}(\mathrm{mm})$}

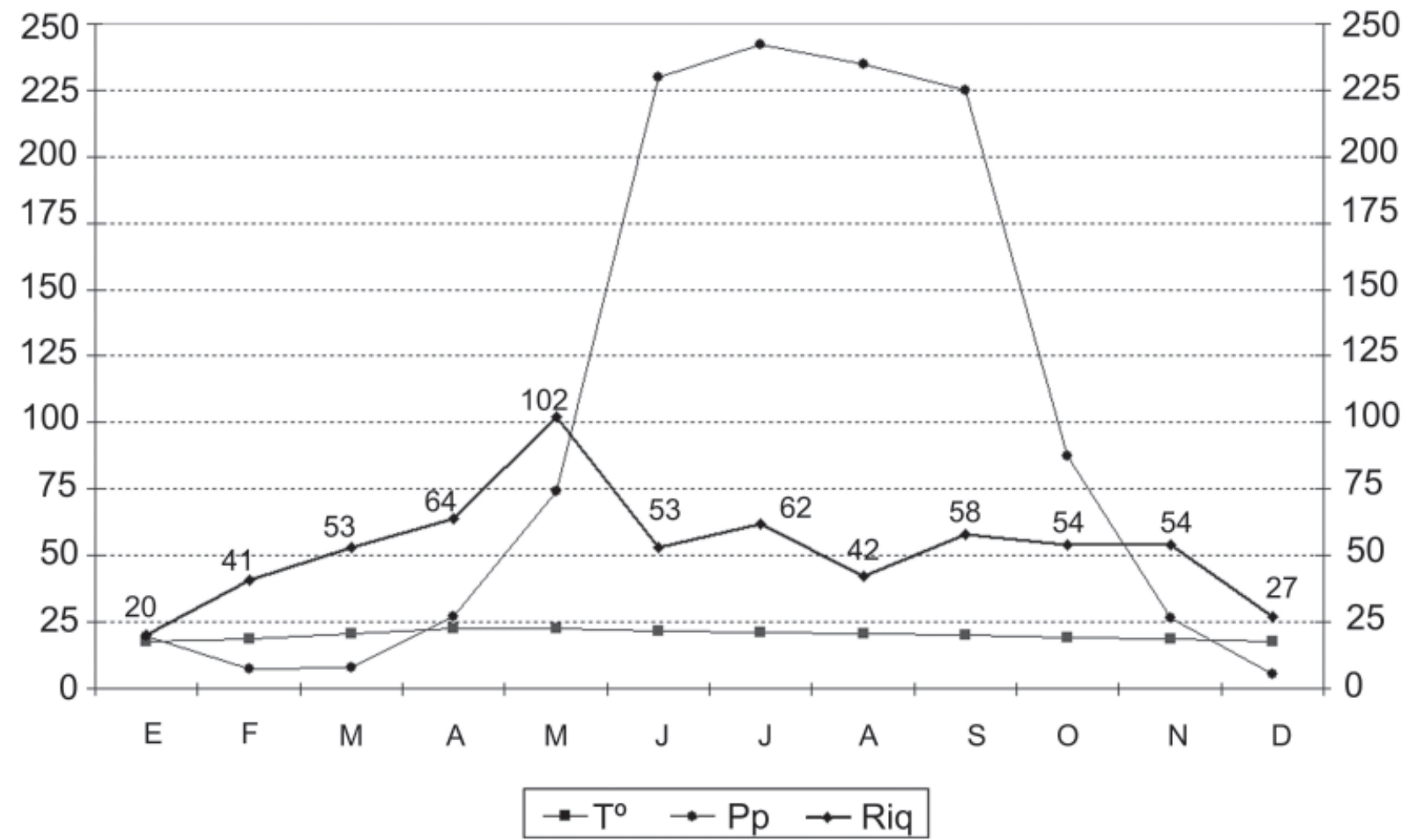

Figura 6. Relación de los meses de vuelo de las especies con los datos climáticos (precipitación y temperatura) citados para la zona por García (1988). $T^{\circ}=$ temperatura, $\mathrm{Pp}=$ precipitación, Riq=número mensual de especies. 
de las mariposas. Con base en los datos de recolecta y las observaciones de campo en los meses de mayor humedad, en julio se registró el valor de riqueza máximo de hesperioideas y papilionoideas (62 spp.) de este periodo, seguido de septiembre (58), junio (53) y agosto (42) (Fig. 6). Durante este tiempo los días de trabajo de campo disminuyeron a 14, debido a que las condiciones metereológicas de nublados y lluvias constantes imposibilitaron el vuelo de los imagos y dificultaron el acceso en algunas localidades.

El cambio estacional en la riqueza de mariposas, también parece estar en función del voltinismo y la sincronización generacional con las condiciones ambientales y nutricionales. De acuerdo con Shapiro (1974), las especies univoltinas tienden a ser monófagas, y las multivoltinas polífagas, por lo que estas últimas pueden emerger en varias épocas del año y tienen oportunidad de sobrevivir, pues sus requerimientos no son tan específicos como en las primeras, cuya emergencia está sincronizada con la época en que sus recursos alimenticios están presentes. La presencia de las lluvias se correlaciona directamente con la abundancia y la riqueza de los insectos (Wolda, 1988), ya que puede afectar la fisiología de la reproducción, el desarrollo ontogenético y la conducta de los imagos; indirectamente también puede afectar a las poblaciones por sus efectos sobre la fenología vegetal. Además, las fórmulas y porcentajes de algunos compuestos presentes en las plantas pueden variar en cada estación y no ser palatables en ciertos meses, por lo que no son aprovechables nutricionalmente por los estadios inmaduros de muchas especies. Esto puede verse reflejado en el tamaño poblacional en la época de no-palatabilidad, o bien, que en esa época sólo se encuentren especies polífagas, pues éstas pueden alimentarse de varias especies o familias de plantas, dado su amplio espectro de recursos alimenticios (Vargas-Fernández et al., 1999).

\section{Agradecimientos}

Al Departamento de Zoología del Instituto de Biología UNAM, por el apoyo técnico para la realización de este proyecto, especialmente a Harry Brailovsky, Cristina Mayorga y Adolfo Ibarra, por su asesoría y por permitirnos el acceso a la Colección Nacional de Insectos, sección Lepidoptera. Al Museo Universitario Dr. Luis Mario Schneider Z., por el apoyo logístico. A Jorge Hernández Ávila, Julia Mejía, Erick Hernández, Elsa Lino y a Luis Antonio Caballero Martínez por su apoyo incondicional en la recolecta de ejemplares. A los proyectos de DGAPA, PAPIIT IN212006 y PAPIME PE200505 y PE201507 que ayudaron al término del trabajo.

\section{Literatura citada}

Adams J. K. 1983. An old first United States record finally published: Papilio victorinus (Papilionidae) in Laredo, Texas. Journal of the Lepidopterists' Society 37:318-318.

Aguilera-Gómez, L. e I. Rivas-Manzano. 2006. Vegetación y flora de Malinalco y su región. In Malinalco y sus contornos a través de los tiempos, X. Noguez-Ramírez (ed.). Colegio Mexiquense, A. C. y Universidad Autonóma del Estado de México, Toluca. p. 25-34.

Arms, K., P. Feeny y R. C. Lederhouse. 1974. Sodium: stimulus for puddling behavior by tiger swallowtail butterflies, Papilio glaucus. Science 185:372-374.

Beutelspacher, C. R. y W. H. Howe. 1984. Mariposas de México. I. Introducción y generalidades. Superfamilia Papilionoidea. La Prensa Médica Mexicana, México, D. F. 128 p.

Clench, H. K. 1979. How to make regional lists of butterflies: some thoughts. Journal of the Lepidopterists' Society 33:216-231.

De la Maza, R. R. 1987. Mariposas mexicanas. Fondo de Cultura Económica, México, D. F. 301 p.

De Vries, J. P. 1987a. The butterflies of Costa Rica and their natural history, vol. I. Papilionidae, Pieridae and Nymphalidae. Princeton Academic Press, New Jersey. 327 p.

De Vries, J. P. 1987b. The butterflies of Costa Rica and their natural history. vol. II. Riodinidae. Princeton Academic Press, New Jersey. 288 p.

Downes, A. J. 1973. Lepidoptera feeding at puddle-margins, dung, and carrion. Journal of the Lepidopterists' Society 27:89-99.

Evans, W. H. 1951. A catalogue of the Hesperiidae indicating the classification and nomenclature adopted in the British Museum (Natural History). Part I. Pyrrhopyginae. British Museum, London, 92 p. 1-9 láminas.

Evans, H. W. 1952. A catalogue of the Hesperiidae indicating the classification and nomenclature adopted in the British Museum (Natural History). Part II. Pyrginae. Section I. London, British Museum. 178 p. 10-25 láminas.

Evans, H. W. 1953. A catalogue of the Hesperiidae indicating the classification and nomenclature adopted in the British Museum (Natural History). Part III. Pyrginae. Section II. British Museum, London. 246 p. 26-53 láminas.

Evans, H. W. 1955. A catalogue of the Hesperiidae, indicating the classification and nomenclature adopted in the British Museum (Natural History). Part IV. Hesperiinae and Megathyminae. British Museum, London. 499 p. 54-88 láminas.

García, E. 1988. Modificaciones al sistema de clasificación climática de Köppen (para adaptarlo a las condiciones de la República Mexicana). Instituto de Geografía, Universidad Nacional Autónoma de México, México, D. F. 220 p.

Godman, F. D. y O. Salvin. 1878-1901. Biologia Centrali Americana. Zoología, Insecta, Lepidoptera Rhopalocera, vols. I-III. Taylor \& Francis, London.

Griffiths, F. J. 1985. Climatología aplicada. Cultural, México, D. F. 154 p.

Guzmán, P. E. 1976. Algunas observaciones sobre lepidópteros 
de Chalma, Estado de México. Revista de la Sociedad Mexicana de Lepidopterología 2:49-51.

${ }^{\S}$ Hernández-Mejía, B. C. 2005. Composición y gremios alimentarios de mariposas diurnas de la superfamilia Papilionoidea (Insecta: Lepidoptera), en el municipio de Malinalco, Estado de México. Tesis Facultad de Ciencias, Universidad Autónoma del Estado de México, Toluca. 99 p.

Howe, H. W. 1975. The butterflies of North America. Doubleday, Garden City, New York. 633 p.

INEGI. 1995. Carta topográfica. 1:50 000. Tenancingo. E14A58. Instituto Nacional de Estadística, Geografía e Informática. México, D.F.

Janzen, H. D. 1967. Synchronization of sexual reproduction of trees within the dry season in Central America. Evolution 21:620-637.

Jenkins, D. 1983. Neotropical Nymphalidae I. Revision of Hamadryas. Bulletin of the Allyn Museum 81:1-146.

Jenkins, D. 1986. Neotropical Nymphalidae V. Revision of Epiphile. Bulletin of the Allyn Museum 101:1-70.

Karlsson, B. 1994. Feeding habits and change of body composition whit age in three nymphalid butterfly species. Oikos 69:224-230.

Karlsson, B. 1995. Resource allocation and mating systems in butterflies. Evolution 49:955-961.

Kendall, R. O. y W. W. McGuire. 1984. Some new and rare records of Lepidoptera found in Texas. Bulletin of the Allyn Museum 86:1-50.

Lamas, G. 1981. La fauna de mariposas de la Reserva de Tambopata, Madre de Dios, Perú. (Lepidoptera, Papilionoidea y Hesperioidea). Revista de la Sociedad Mexicana de Lepidopterología 6:23-40.

Llorente-Bousquets, J., A. Luis-Martínez e I. Vargas-Fernández. 1990. Catálogo sistemático de los Hesperioidea de México. Publicaciones Especiales del Museo de Zoología, Facultad de Ciencias, Universidad Nacional Autónoma de México, México, D.F. 1:1-70.

Llorente-Bousquets. J., L. Oñate, A. Luis-Martínez e I. VargasFernández. 1997. Papilionidae y Pieridae de México: distribución geográfica e ilustración. Comisión Nacional para el Conocimiento y Uso de la Biodiversidad y Facultad de Ciencias, Universidad Nacional Autónoma de México, México, D. F. 227 p. 28 láminas.

Llorente-Bousquets J., A. Luis-Martínez e I. Vargas. 2006. Apéndice general de Papilionoidea, Distribución Estatal y Provincias Biogeograficas. In Componentes bióticos principales de la entomofauna mexicana, J. J. Morrone y J. Llorente-Bousquets (eds.). Las Prensas de Ciencias, Facultad de Ciencias, Universidad Nacional Autónoma de México, México, D.F. p. 733-792.

\$Luis-Martínez, A., J. Llorente-Bousquets, I. Vargas y A. D. Warren. 2003. Biodiversity and Biogeography of Mexican butterflies (Lepidoptera: Papilionoidea and Hesperioidea). Proceedings of the Entomological Society of Washington 105:209-224.

Luis-Martínez, A., J. Llorente-Bousquets e I. Vargas-Fernández. 2003. Nymphalidae de México I (Danainae, Apaturinae, Biblidinae y Heliconiinae): Distribución geográfica e ilustración. Comisión Nacional para el Conocimiento y Uso de la Biodiversidad y Facultad de Ciencias, Universidad Nacional
Autónoma de México, México, D. F. 249 p. 30 láminas.

Luis-Martínez, A., J. Llorente-Bousquets e I. Vargas. 2005. Una megabase de datos de mariposas de México y la regionalización biogeográfica. In Regionalización biogeográfica en Iberoamerica y tópicos afines: Primeras Jornadas Biogeográficas de la Red Iberoamericana de Biogeografía y Entomología Sistemática (RIBES XII. I-CYTED), Llorente-Bousquets, J. y J. J. Morrone (eds.). Las Prensas de Ciencias, Facultad de Ciencias, Universidad Nacional Autónoma de México, México, D.F. p. 269-294.

Miller, L. D. 1974. Revision of the Euptychiini (Satyridae). 2. Cyllopsis R. Felder. Bulletin of the Allyn Museum 20:1-98.

Miller, L. D. 1978. Notes and descriptions of Euptychiini (Lepidoptera: Satyridae) from the Mexican region. Journal of the Lepidopterists' Society 32:75-85.

Miranda, F. y E. Hernández-Xolocotzi. 1963. Los tipos de vegetación de México y su clasificación. Boletín de la Sociedad Botánica de México 28:29-179.

Morón, M. A. y R. A. Terrón. 1988. Entomología práctica. Instituto de Ecología, Xalapa, Veracruz, México. 504 p.

Murphy, D. D., A. E. Launer y P. R. Ehrlich. 1983. The role of adult feeding in egg production and population dynamics of the checkerspot butterfly Euphydryas editha. Oecologia 56:257-263.

Pozo, C., J. Llorente-Bousquets, A. Luis-Martínez, I. VargasFernández y N. Salas Suárez. 2005. Reflexiones acerca de los métodos de muestreo para mariposas en las comparaciones biogeográficas. In Regionalización biogeográfica en Iberoamerica y tópicos afines: Primeras Jornadas Biogeográficas de la Red Iberoamericana de Biogeografía y Entomología Sistemática (RIBES XII.I-CYTED), J. LlorenteBousquets y J. J. Morrone (eds.). Las Prensas de Ciencias, Facultad de Ciencias, Universidad Nacional Autónoma de México, México, D.F. p. 203-215.

Reyes, T. C. 1992. Recursos Hidrológicos. In Atlas del Estado de México, M. A. García, L. Mendoza, N. Pineda, S. Franco, M. Ocampo, A. Saker, R. Franco, J. Oscos y S. Salazar (eds.). Gobierno del Estado de México y Universidad Autónoma del Estado de México. Toluca. p. 85.

Rydon, A. 1964. Notes on the use of butterfly traps in east Africa. Journal of the Lepidopterists' Society 18:51-58.

Schneider, L. M. 1999. Malinalco: Monografía Municipal. Instituto Mexiquense de Cultura, Gobierno del Estado de México. Toluca. p. 93

Scott, A. J. 1986. The butterflies of North America. A natural history and field guide. Stanford University Press, California. $583 \mathrm{p}$.

Seitz, A. 1907-1924. The macrolepidoptera of the world, vol. V. 8. Alfred Kernen, Stuttgart.

Sevastopulo, D. G. 1974. Lepidoptera feeding at puddle-margins, dung and carrion. Journal of the Lepidopterists' Society 28:167-168.

Shapiro, M. A. 1974. The butterfly fauna of the Sacramento Valley, California. Journal of the Lepidopterists' Society 13:73-82.

Vargas, I. F., J. B. Llorente-Bousquets y A. Luis-Martínez. 1992. Listado lepidopterofaunístico de la sierra de Atoyac de Álvarez 
en el estado de Guerrero: notas acerca de su distribución local y estacional (Rhopalocera: Papilionoidea). Folia Entomológica Mexicana 86:41-178.

Vargas, I. F., J. Llorente-Bousquets y A. Luis-Martínez. 1999. Distribución de los Papilionoidea (Lepidoptera: Rhopalocera) de la sierra de Manantlán (250-1650 m snm) en los estados de Jalisco y Colima. Publicaciones Especiales 11, Museo de Zoología, Facultad de Ciencias, Universidad Nacional Autonóma de México, México, D. F. 153 p.

Vázquez, L. G. 1951. Observaciones sobre piéridos mexicanos II. Phoebis sennae eubule y sus formas en México. Anales del Instituto de Biología, Universidad Nacional Autónoma de México, México, D.F. 21:417-429.

Vázquez, L. G. y H. Pérez. 1962. Observaciones sobre la biología de Baronia brevicornis Salvin. (Lepidoptera:
Papilionidae-Baroniinae). Anales de Instituto de. Biología, Universidad Nacional Autónoma de México, Serie Zoología 32:295-311.

Warren, A. D. 2000. Hesperioidea (Lepidoptera). In Biodiversidad, taxonomía y biogeografía de artrópodos de México: hacia una síntesis de su conocimiento, vol. II, J. Llorente-Bousquets, E. González y N. Papavero (eds.). Facultad de Ciencias, Universidad Nacional Autónoma de México. p. 535-580.

Wolda, H. 1988. Insect seasonality: why? Annual Review of Ecology and Systematics 19:1-18.

Young, A. M. 1975. Feeding behavior of Morpho butterflies (Lepidoptera: Nymphalidae: Morphinae) in a seasonal tropical environment. Revista de Biología Tropical 23:101-123. 